Oikos 121: 1966-1976, 2012

doi: $10.1111 / j .1600-0706.2012 .20184 . x$

(C) 2012 The Authors. Oikos (C) 2012 Nordic Society Oikos

Subject Editor: Daniel Gruner. Accepted 13 February 2012

\title{
Daily foraging cycles create overlapping time-scales in functional responses
}

\author{
Jérôme Casas and Edward McCauley \\ J. Casas (casas@univ-tours.fr), Inst. de Recherches sur la Biologie de l'Insecte, Univ. de Tours, IRBI UMR CNRS 7261, Av. Monge, FR-37200 \\ Tours, France. - E. McCauley, Dept of Ecology, Evolution and Marine Biology, Univ. of California at Santa Barbara, Santa Barbara, \\ CA 93106-9620, USA. Present address: Dept of Biological Sciences, Univ. of Calgary, Calgary, AB, T2N 1N4, Canada.
}

\begin{abstract}
The functional response is one of the most widely measured attributes of consumers. Phenomenalistic descriptions of how predator attack rates vary with prey density are fundamental components of consumer-resource models. The application of these functions typically assumes continuous foraging by individuals, along with stationarity in their behavioural and physiological processes. Yet most species display a diurnal cycle in foraging and resting, and the impact of this foraging pattern on the functional response is unknown.

We use a physiologically structured or 'state-space' approach to examine how the daily foraging cycle affects the temporal dynamics of attack rates and the functional response of parasitoids (Aphytis melinus) and predators (Mantis crassulea and fishes). The state spaces for parasitoids and predators are the number of mature eggs, the eggload, and the satiation level, respectively. The corresponding rates are those of egg maturation and oviposition on one hand, and digestion and prey capture on the other hand.

We show that the length of the foraging period alters both the shape of the functional response and the magnitude of attack rates, compared to a daily functional response proportional to the time spent foraging, as is classically done. Our models reveal how separation of time-scales arises between behavioural or physiological and lifetime processes, and the difficulty in resolving such separation once the new time scale of a foraging cycle is introduced.

Foragers in many environments, either because of exposure to low resource density or constrained by short foraging windows, cannot achieve the behavioural or physiological stationarity assumed in classical analyses. This introduces a fundamental mixture of time-scales that has significant effects on estimates of attack rates. Using a population-level model of predators and prey, we show how restricted periods of daily foraging have important dynamical consequences both in terms of equilibrium levels and return times to these equilibria.
\end{abstract}

The functional response of predators, parasitoids, and more generally of consumers, has been studied for a large number of species (extensively reviewed by Jeschke et al. 2002, Keeling et al. 2000, Fernandez-Arhex and Corley 2003, Hobbs et al. 2003, Fryxell et al. 2007, Tschanz et al. 2007, Englund and Leonardsson 2008). Behavioral ecologists are fascinated by how individuals search, detect and capture prey as the density of these resources varies in quantity or quality in different environmental settings. Physiological ecologists use these relationships to understand energy acquisition and allocation, thereby linking physiological processes to resource levels in the environment. Population and community ecologists use the functional response to infer mortality rates imposed on prey, and also as a key component to dynamically couple prey and predator populations in models of predator-prey systems or foodwebs (Fryxell and Lundberg 1997, Murdoch et al. 2003, Turchin 2003). The study of functional responses thus spans many levels of biological organization and environmental settings.
Integrating the information across levels of biological organization (i.e. from physiological to individual action, or across individuals to population-level responses) in these different environmental settings to construct functional responses presents some significant challenges, especially when these relationships are subsequently used to understand the dynamics of predator and prey populations operating over larger spatial and temporal scales. For example, individual predators often forage over landscapes or in environments where there is significant spatial variation in quantities of prey, and recent work has examined how this spatial variability alters the functional response and hence the interaction rates between predators and prey in space (Morozov 2010). While spatial variation has received much attention, there is surprisingly little research on the effects of temporal changes in prey density on the functional response. This temporal variation can arise in a variety of ways. Indeed, many predators possess diel or diurnal foraging patterns, and several other chronobiological rhythms are of great importance in the life of many organisms (Dunlap et al. 2009). 
For example, the foraging window of a leafminer parasitoid is set by a complex interaction of environmental (day/night cycles) and biotic (plant volatiles emitted after herbivore's attacks), the latter being also driven by the day/night shifts (Zhang et al. 2010). Ruminants, wild and domesticated, also have complex diurnal patterns of grazing, alternating between active and resting periods, with consequences for metabolism (Gregorini et al. 2008, Kramer and Prins 2010). Thus, foraging periods for many consumers are punctuated by periods of inactivity - either because of physiological, behavioral, or environmental constraints, operating singly or in combination. In this paper, we show that temporal variation in foraging activity can have profound effects on the estimate of interaction rates for predator-prey dynamics.

The typical approach used to incorporate the functional response of predators into dynamic models of resourceconsumer systems is to assume continuous and instantaneous functions. Simply put, the instantaneous attack rates are measured at different prey densities in short-term behavioral experiments, either in the lab or field, and are then scaled up proportionately to the appropriate foraging period and used to parameterize dynamic functions in population-level models (Abrams 1982, Owen-Smith 2002). For example, rates measured at the scale of minutes are extrapolated to a daily period. We refer to rates calculated from this approach as derived from a proportional model. There have been many studies that have criticized the experimental design used to infer these rates (e.g. prey depletion during the observational period, arena size limitation), and demonstrate how biases might emerge (summarized by Fellows et al. 2007). While these criticisms are important and eliminating these biases would lead to more accurate estimates of the instantaneous attack rates and their dependence on the quantity of prey, there remains the fundamental problem how to integrate these instantaneous rates over the appropriate time-scales of the dynamics of the predator-prey interaction. The simple approach that is most commonly used implicitly assumes that the functional response is time-invariant, and hence that all underlying behavioral and physiologically processes are constant.

In a benchmark series of papers, Metz and van Batenburg developed a mechanistic approach that takes into account the dynamics of the underlying physiological and behavioral processes to yield the functional response for a predator (Metz and van Batenburg 1985a, b). This was stimulated by the original experimental work by Holling on mantids (Holling 1966). Here, we use elements of this approach to study the dynamic consequences of variation in the length of the foraging period. The Metz' 'state-space approach' considers a population of individual predators represented as a function of individual physiological states (Fig. 1.1). Satiation, often equated to gut content in empirical studies, is the state variable used to model the functional response of predators. Satiation increases in jumps, reflecting the capture of prey items, and decreases smoothly during digestion. Behavioural activities, such as prey capture and handling, are treated as a part of the prey capture rate. Prey capture rates depend on prey density, but digestion rate does not (Fig. 1.1). Thus, changes in satiation of the predator population over time can be represented by a stochastic mixed continuous/jump Markov PDE equation, in which the continuous part is wholly deterministic (Fig. 1.2). We will call this physiologically structured model the full model.

The analytical resolution of this full model is highly complex, requiring different analytical limit processes to determine the predator stationary satiation distribution at different prey density (Fig. 1.3, 1.4). Stationarity, or steady state, refers here to an equilibrium distribution of states resulting from the asymptotic behavior of a stochastic process (Hunter 1983). Letting time go to infinity has major advantages. Given sufficient time, the predator population reaches stationarity through the interplay of behavioural activities, such as prey pursuing and attacking, and physiological processes, such as digestion (i.e. solving for the equilibrium distribution from the PDE - Fig. $1.2 \rightarrow 1.4$ ). The mean value of the predator satiation level is then used in a final step to compute the mean number of prey eaten, based on the prey capture rate at a given level of satiation. Alternatively, the model is first simplified by assuming that predators are sucking a broth of tiny, nearly weightless and numerous prey, thus smoothing out the stochastic upward jumps. The stochasticity inherent to prey capture is lost, with the deterministic approximation (Fig. 1.3) becoming equivalent to the mean value of the stochastic process. The functional response is subsequently obtained by varying the prey density. This approach, to varying degrees, has been previously applied to spider mites, Daphnia, mantids, predatory bugs, fishes and host-parasitoid systems (Sabelis 1986, Mangel and Peters 1989, Gurney et al. 1990, Hall et al. 1995, Briggs et al. 1995, Van den Meiracker and Sabelis 1999, Rijn et al. 2005). In summary, although intrinsically more realistic due to consideration of the underlying physiological and behavioural processes, the full model, once time is taken to infinity, shares the same major limitation displayed by the proportionality rule: time within a foraging bout has been factored out, such that stationarity is assumed at any instant.

The derivation of functional responses used in consumerresource models is well known, and can be found in virtually every undergraduate ecology textbook. However, once these equations describing the functional response (e.g. Holling's disc equation or the Michaelis-Menten equation) are used in dynamic predator-prey models, implicit assumptions about the time-scale of the behavioural response by predators and the rate of change of the prey population are then introduced. In essence, sub-populations of handling and searching predators reach a 'pseudo-equilibrium' on fast time scales (Metz and van Batenburg 1985a) to yield an attack rate by the average predator for that instantaneous density of prey. By not assuming this pseudo-equilibrium, we can demonstrate the impacts of cyclic foraging periods on the equilibrium and stability of predator-prey systems. The full model (Fig. 1.1, 1.2), without taking the limits, enables us to investigate numerically these implications of temporal variation on estimates of the functional response.

The implications of cyclic interruptions of foraging for physiologically structured populations are indeed not clear. Some processes directly related to predation and parasitism are active over the entire daily cycle, such as digestion in predators and egg maturation in parasitoids. Interruptions would therefore be expected to have complex effects on population frequency distributions within their physiological state space 


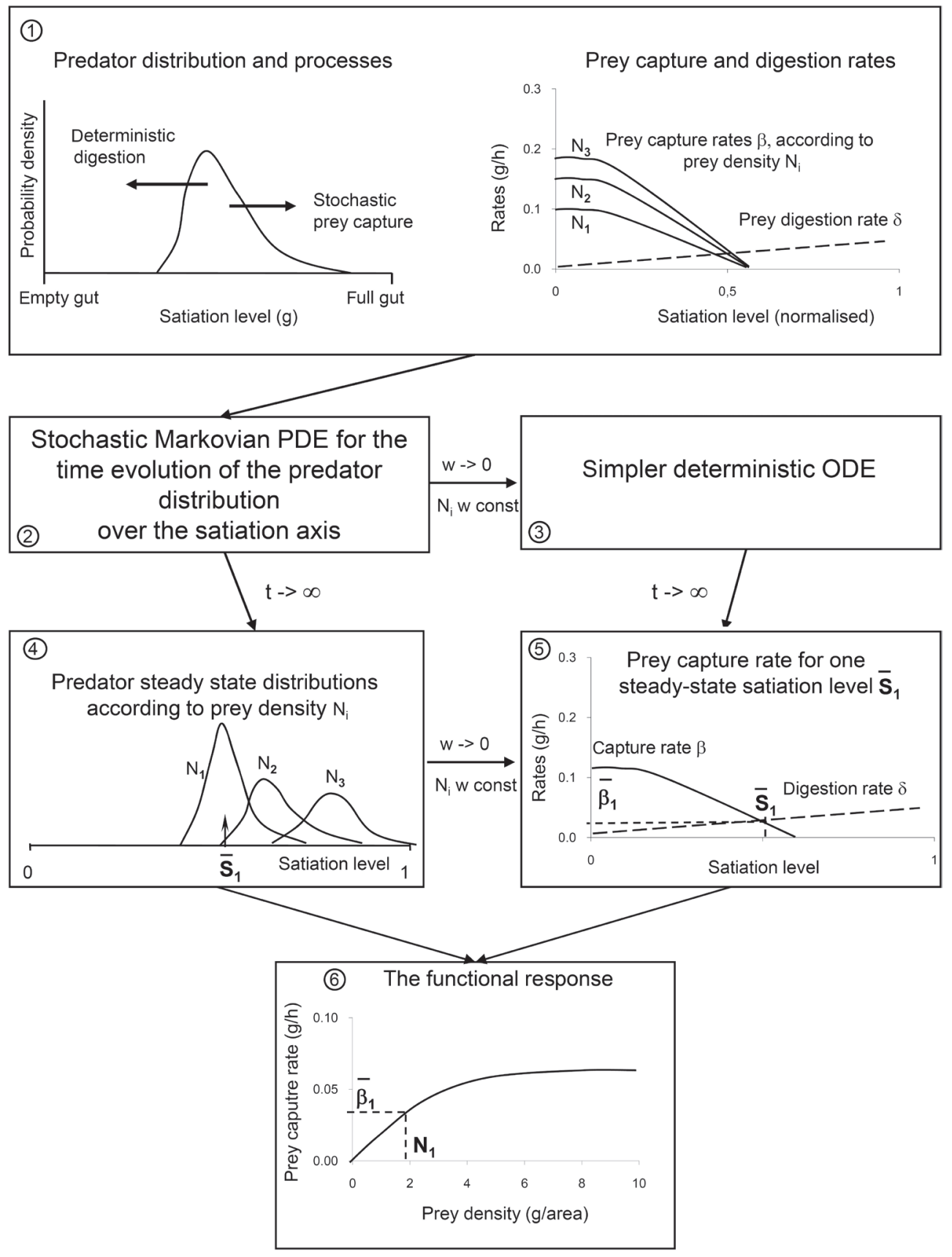

Figure 1. The functional response of predators based on the physiologically structured population modelling approach. The predator population is represented by a distribution in a one dimensional state space, satiation. Satiation level, or gut fullness, is expressed in body weight (g). Downward movements of the predator population are due to digestion and upward movements are due to prey capture (panel 1). The prey size, which defines also the upwards step size, is $\mathrm{w}(\mathrm{g})$. Both prey capture and digestion are a function of satiation. Prey capture, in contrast to prey digestion, is also dependent on prey density ( $\mathrm{Ni}$, expressed in weight $\left(\mathrm{g} \mathrm{area}^{-1}\right)$ for consistency). The full stochastic partial differential equation (PDE) (panel 2) describing the movement of the distribution of predators over time can be simplified to a deterministic ordinary differential equation (ODE) through several assumptions (panel 3). See text for more information about the different assumptions. For a given prey density, the value of the approximated deterministic predator satiation level at equilibrium (panel 3) or the (mean) value of satiation of the stationary predator distribution (panel 4) is then used to compute the prey capture (panel 5). The functional response is then generated by varying prey density (panel 6).

on a daily time scale. These effects may ripple further up to the lifetime scale and up to the population dynamics level, in case the predation rate is strongly modified. Our specific aim is therefore to obtain the most realistic model possible of the functional response, taking into account the daily cycles of foraging, by developing a full model and numerically computing changes in the consumer population over time for different foraging windows. This enables us to compare the functional response predicted using the full model with the response predicted using the proportional model. Cyclical interruptions being present in both approaches, the comparison of the predation rates are for the same amount of 
time available for foraging, but based on different underlying processes.

Our more general goal is to examine how the length of the daily foraging period affects the estimation of functional response curves, and the implications for modelling the dynamics of predator-prey systems. We use three examples of organisms with strikingly different biology to illustrate the generality of our results. Our first example uses the parasitoid Aphytis melinus as a model organism to develop the entire approach. Indeed, it is one of the very few species for which quantitative information is available on all parameters: the activity window in the field, the physiological and behavioural processes determining egg maturation and oviposition, and the role of these processes in the population dynamics and control of its host pest, the California red scale (Casas 2000, Casas et al. 2000, 2004, Murdoch et al. 2003 , 2005). Such information is only partially available for other parasitoids, predators and herbivores. We further developed the approach in fewer details for two predators, the well known Mantis crassulea example of Holling (1966, Metz and van Batenburg 1985a, b) and a fish, whiting Merlangius merlangus (Hall et al. 1995). Thus, our examples span from terrestrial ambushing invertebrate predators and active searching parasitoids, for which digestion and egg maturation are the physiological processes of importance, respectively, and a vertebrate aquatic predator.

\section{Methods}

\section{Modelling parasitoids}

Eggload is the relevant physiological state variable when working with parasitoids (Fig. 2A), as it determines the attack behaviour fundamental to the functional response

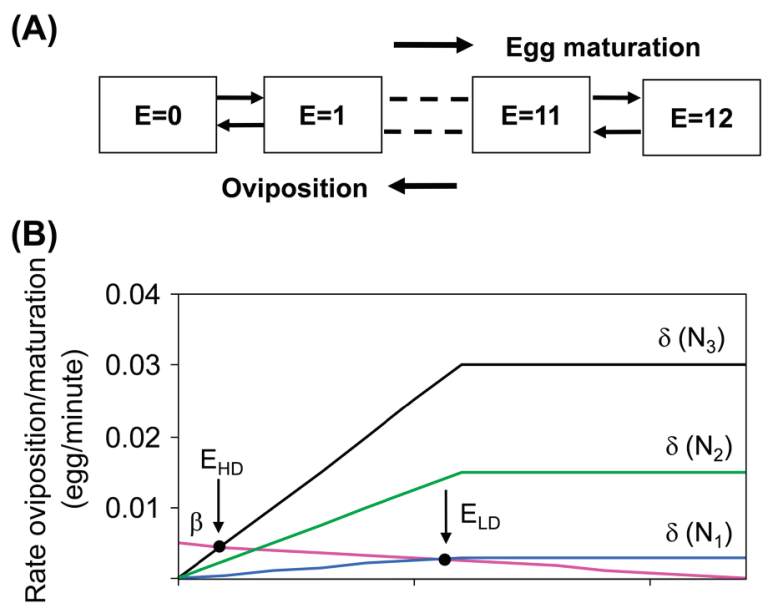

Figure 2. State space and process rates for modelling physiologically structured Aphytis parasitoid populations. (A) The state variable eggload and the possible transitions in the state space for the parasitoid Aphytis melinus. (B) Eggload-dependent egg maturation (pink) and oviposition rates at high (black), intermediate (green) and low (blue) host densities. The mean values of predicted stationary populations for an infinite foraging window at high $\left(\mathrm{E}_{\mathrm{HD}}\right)$ and low $\left(\mathrm{E}_{\mathrm{LD}}\right)$ host densities are indicated (parameter values of host densities are: high host density $=1$, intermediate $=0.5$ and low host density $=0.1$ ).
(Casas et al. 1993). Eggload is a discrete state variable and accounts only for mature eggs. Egg maturation is associated with moving up in the state space, and is equivalent to prey capture for predators; oviposition implies moving down in the state space, equivalent to prey digestion. The parasitoid population is modelled by a (non-linear) birth and death cycling markovian chain model, with maxima at the lowest and highest eggloads and parameters varying periodically with time (Parthasarathy and Lenin 2004).

Such a model requires the birth and death rates, and the time periods in which different rates operate, as input. Each of these parameters is treated in the following. Egg maturation occurs over the whole cycle and is modelled as a birth process. The rate of this activity $(\beta)$ was assumed to be a linear decreasing function of eggload (E), according to previous experimental studies on Encarsia formosa (van Vianen and van Lenteren 1986) and statistical model fitting on A. melinus (Casas et al. 2000). The maximal value of the egg maturation rate was obtained earlier $\left(\beta_{0}=0.0048\right)$ for eggless females (Casas et al. 2000). Oviposition, modelled as a death process, only occurs during periods of foraging. The rate of this activity $(\delta)$ is linearly dependent on eggload until each of the six ovarioles is processing its mature egg, at which point a plateau is reached (Fig. 2B). The monotone increase in the oviposition rate is most likely due to the increased acceptance of hosts with increasing eggload or increased searching activity (Casas et al. 2000). The mechanisms for the changing motivation can be purely physiological and do not need any variability in host quality. The motivation evolves in a way opposite to the declining tendency to host-feed (feeding on the host fluids after puncturing with the ovipositor) with increasing eggload (Collier 1995, Collier et al. 1994). The rate of oviposition is highest under conditions of 1) high density of the preferred host instar (negligible searching time and highest acceptance rate) and 2) maximal eggload. We previously observed a maximal egglaying rate of 0.026 eggs/ min under such conditions in the laboratory (Casas et al. 2004). It is very similar to the rate observed in another study (0.03 eggs $\left.\mathrm{min}^{-1}\right)$, conducted in the field, under similar conditions of high host density (Casas et al. 2000). Thus, we used the higher rate as the maximal possible oviposition rate for six to 12 mature eggs, the latter figure being the highest eggload. The foraging time window varies between three and eight hours in the field, with the active period beginning late morning (Casas 2000, Murdoch et al. 2005). Short resting spells within this window are included, so that a $24 \mathrm{~h}$ day is composed of a single foraging period and the following night.

Let us define the probability $P_{i}(t)$ of eggload $i$ at time $t$ as the fraction of the population that has $i$ mature eggs at time $t, \delta_{i}$ as the oviposition rate, $\beta_{i}$ as the egg maturation rate, both at eggload $i$, and $m$ the highest egg complement carried by a female. The birth-death equation determining the dynamics of eggload distribution is therefore:

$$
\begin{array}{ll}
\frac{d P_{i}}{d t}=-\left(\delta_{i}+\beta_{i}\right) P_{i}+\beta_{i-1} P_{i-1}+\delta_{i+1} P_{i+1} & \text { for } i=1, \ldots m-1 \\
\frac{d P_{0}}{d t}=-\beta_{0} P_{0}+\delta_{1} P_{1} & \text { for } i=0
\end{array}
$$


$\frac{d P_{m}}{d t}=\beta_{m-1} P_{m-1}-\delta_{m} P_{m} \quad$ for $i=m$

To compute the functional response, we used the fundamental mass action postulate stating that the oviposition rate is the product of the host density times the 'rate constant of effective prey encounters' (Metz and van Batenburg 1985a). We scaled host density by defining the lowest host density, at which the maximal oviposition rate $\left(\delta_{\mathrm{I}}=0.03, i=6 \ldots 12\right)$ is attained, as 1 . Lower host densities lead to a proportional decrease in the oviposition rate; higher host densities do not lead to further increases in oviposition rate.

For the full model, the functional response is estimated by summing up all laid eggs during a given foraging time window (i.e. all $\left.\delta_{i} P_{i}(t)\right)$. For the proportional model, the functional response is based on that obtained using the full model firstly run with a foraging window of $24 \mathrm{~h}$ (i.e. no cyclicity). It is then divided proportionally to match the length a given foraging window. All computations were done in MATLAB (Mathworks), in particular using the matrix algebra function expm for computing the transient distributions.

\section{Modelling predators}

The description of the models for predators is succinct, given that the same approach as for Aphytis was used. We first developed a model for Mantis crassulea, the species for which the functional response has been most thoroughly analysed by Holling (1966). The original model of Holling contains some 14 parameters, reduced to half in simplified model developed by Metz and van Batenburg (1985a, b), within the physiologically structured framework. We followed the same modelling approach as Metz and van Batenburg, using the parameters values and the prey densities given in the above papers. The satiation level was discretized in 38 units, required in the birth-death processes approach, a single unit representing the weight of a single prey. Figure 3 clearly shows that the prey capture rate is much higher than the digestion rate, except for the lowest values of prey density.

The approach was also applied to fishes: whiting Merlangius merlangus, and a generic fish with very similar parameter values (Hall et al. 1995). Some values were unfortunately not reported or available in this study. While the digestion rate $\left(0.15 \mathrm{~g} \mathrm{~h}^{-1}\right.$, their Table 2$)$, the foraging window (from 3 a.m. to 10 a.m.) as well as the capture rate $\left(0.25 \mathrm{~g} \mathrm{~h}^{-1}\right.$, their Fig. 13) are given for whiting, the later is not a function of the state of the animal, but assumed to be a constant. We also assumed, on the basis of their Fig. 6, that a prey item weights $0.25 \mathrm{~g}$. Given these conditions, the capture and digestion rates cross when the fish is near to starvation level. We do not have functional response data to compare with our predictions, but there is an estimate of the daily food intake of whiting (their Table 5) as well as the time history of the mean gut weight, assuming infinite foraging window, for the model fish with similar rates as whiting (their Fig. 6A).

As done previously for Aphytis, the proportional model for both predators is first obtained using the full model with a $24 \mathrm{~h}$, hence infinite, foraging window. The functional

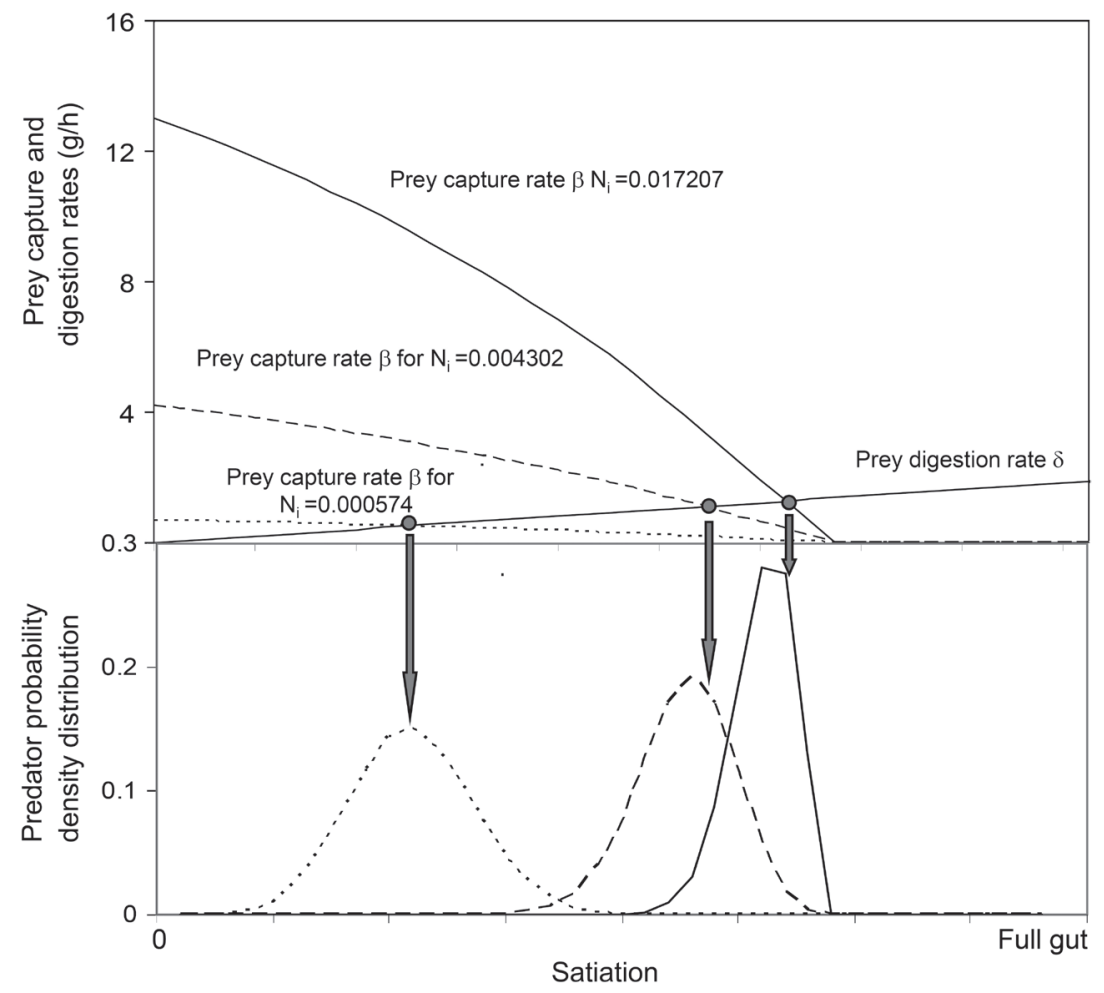

Figure 3. Process rates for modelling physiologically structured Mantis predator populations and long term equilibrium distributions. Rates of prey capture and digestion for Mantis crassulea (top graph) and predator satiation distribution for the three prey densities at equilibrium for a $24 \mathrm{~h}$ foraging period (bottom graph). The predator distribution is a histogram, as the continuous satiation space variable has been discretized for the need of computation. The mean satiation level of the predator distribution at equilibrium is highlighted. 
response is then scaled proportionally down to the required foraging window.

\section{Results}

\section{Parasitoid population distribution and their functional response}

Figure $4 \mathrm{~A}$ shows the predicted distribution of parasitoid states at the beginning and end of an 8-h foraging period from our model. Recall that eggload is the balance between the maturation and oviposition rate. Note the large change in the distribution of parasitoid states in the presence of high host density compared to low host density. The 8-h period is the maximum observed for Aphytis. Figure $4 \mathrm{~b}$ shows how the mean eggload (i.e. the average of the distribution of predator states) varies over the entire range of foraging periods (e.g. 3-8 h). Figure $4 \mathrm{~B}$ also shows the equilibrium values that would be obtained from an infinite foraging period at low and high host densities, labelled $\mathrm{E}_{\mathrm{LD}}$ and $\mathrm{E}_{\mathrm{HD}}$, respectively. The parasitoid population distribution did not achieve steady-state values over most of the foraging time windows, except at high host density and at the end of a long period of foraging (Fig. 4B). At high host densities, females lay most of their ripe eggs within a couple of hours. The number of eggs laid each day thereafter reached a plateau determined by the

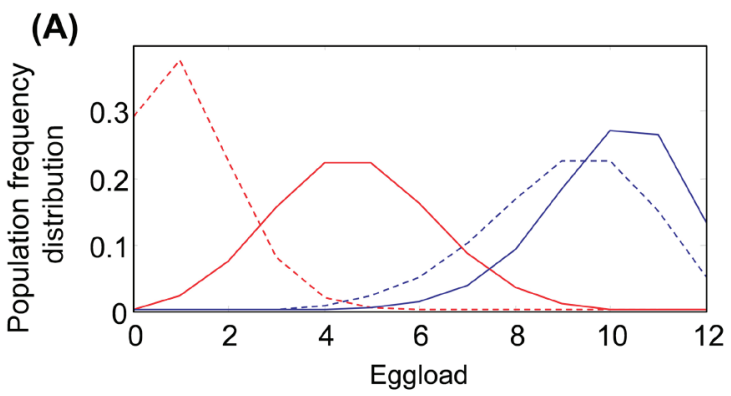

(B)

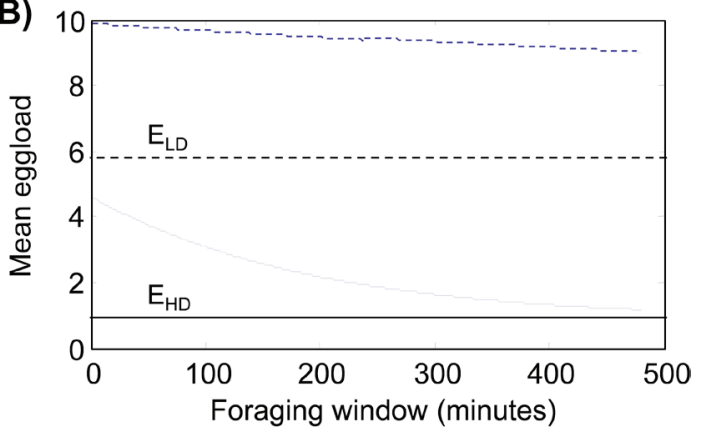

Figure 4. Aphytis parasitoid population distributions at the beginning and end of a foraging period and daily dynamics of the eggload. (A) The predicted population eggload frequency distribution at the beginning (full lines) and end (dotted lines) of an eight hour foraging window for high (red) and low (blue) host densities. (B) Mean eggload during a foraging window of eight hours for low (dotted line) and high (full line) host densities. Starting and final eggload distributions are given in Fig. 4A. The mean eggload for an infinite foraging window at high and low host densities are given by the lines $\mathrm{E}_{\mathrm{HD}}$ and $\mathrm{E}_{\mathrm{LD}}$, as in Fig. 2 (parameter values of host densities are high host density $=1$ and low host density $=0.1$ ). egg maturation rate, as females cannot lay eggs which have not been produced. At low host densities, females lay eggs more slowly, and will not reach the equilibrium value even during an unlimited, i.e. $24 \mathrm{~h}$ continuous foraging period $\left(\mathrm{E}_{\mathrm{LD}}\right.$, Fig. 4).

The impact of these processes has a major effect on the functional response of the parasitoid (eggs laid per foraging window), which can be observed by evaluating the full model over a range foraging periods and host densities, and comparing expectations from the proportional model. Increasing foraging time produces a marked shift from linear to hyperbolic functional responses (Fig. 5A). The proportional model strongly underestimates the true number of hosts attacked during a foraging window (Fig. 5B).

\section{Applying the approach to predators}

The overall results and messages for both predators are the same as for Aphytis. A comparison of the predictions of the functional response of Mantis from our full model with those of Holling's original model, as well as a discussion of the small discrepancies, is given in Appendix 1. Figure 6 shows that the proportional model systematically underestimates the attack rate of Mantis at all prey levels and for any length of the foraging window. The functional response
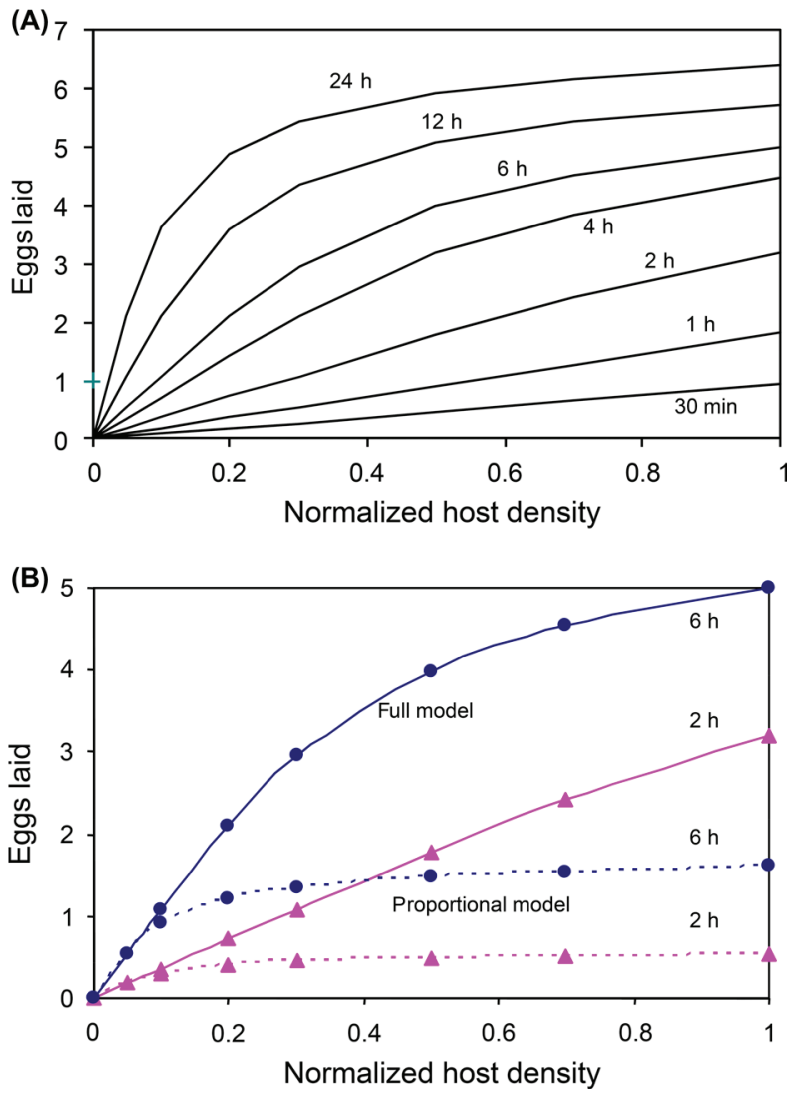

Figure 5. Functional responses for Aphytis parasitoids. The functional response predicted by the stochastic model as a function of time spent foraging each day (A) and comparison (B) between the functional responses predicted by the stochastic model (full lines) and the proportionality rule (dotted lines) for foraging windows of 2 and 6 h ( $8 \%$ and $25 \%$ of a day; pink triangles and blue dots, respectively). 


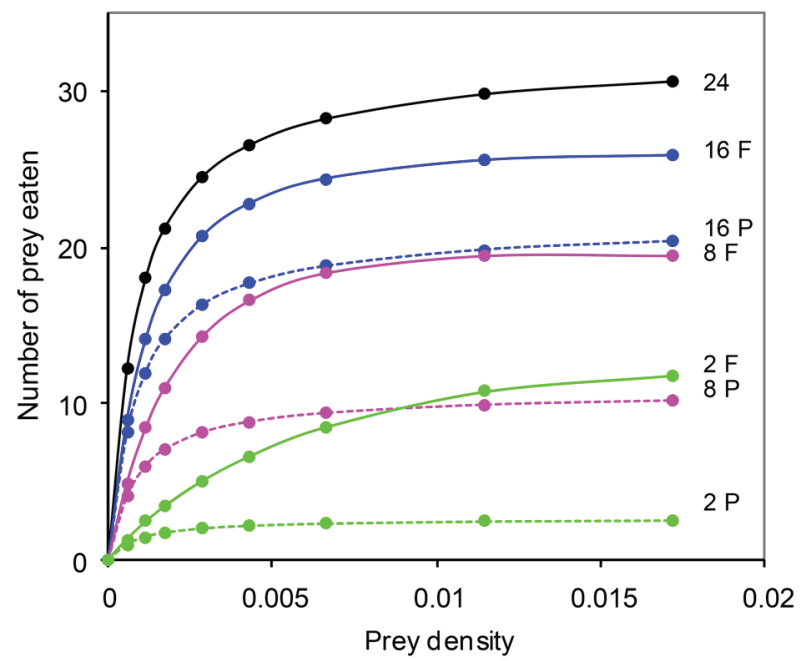

Figure 6. Comparison of the full (F) and proportional (P) models for Mantis crassulea for foraging windows of 2, 8 and $16 \mathrm{~h}$.

derived from the full model shows the characteristic hyperbolic shape, and decreasing the foraging window reduces the curvature, as few predators become satiated when the foraging period is short. The full model run for the fish model species analysed in Hall et al. (1995, their Fig. 6) predicts for an infinite foraging window 1) a mean satiation level of 0.48 $\mathrm{g}$, which compares quite favourably with $0.5 \mathrm{~g}$ estimated from Fig. 6A of Hall et al. (1995), and 2) a daily weight gain of $1.75 \mathrm{~g}$, as predicted by the above authors.

\section{Discussion}

\section{Generality of the approach}

The similarities in the results obtained with three strikingly different examples illustrates the generality of the approach. For predators, as for parasitoids, the state dependent rate of resource acquisition is broadly inverse to the rate of resource processing. This leads to a stable equilibrium if the two processes operate over a long time horizon. A day is however split into two major periods. During the foraging period, both processes are present, moving the consumer distribution towards that equilibrium. During the resting period however, the slower process is the only active one. It moves the distribution away from the equilibrium, towards one of the extremes of the state space. At the onset of the next foraging period, the quicker process brings the distribution back towards the equilibrium point. The resting time is thus preparing the next foraging period as the consumer distribution moves in a region of the internal state space which ensures high rate of consumption at the onset of the next foraging region. Both periods are thus intimately connected through the constraint of adding up to $24 \mathrm{~h}$; the end of a foraging period is thus not a mere interruption by the incoming night.

A variety of mechanisms produces foraging windows, and more generally varying activity levels. They can be related to the behaviour of the predator or the prey, including for example migratory patterns, risk sensitive foraging etc. (see for example Joern et al. 2006 for a temperature-dependent overlap of activity windows between spiders and grasshoppers) or they could be externally imposed via extrinsic environmental variation (e.g. tidal cycles, as in some of the fishes studied by Hall et al. 1995). In addition, the length of the foraging window might depend on the state of the predator, inducing a feedback loop, as for example in common eiders which expand their foraging window greatly in winter in order to maintain energy balance (Guillemette 1998).

The importance of these swings and transient periods will be a function of five variables: the relative extents of the foraging and resting periods, the relative and absolute speeds of the processes, the span between the equilibrium position and the extreme state attained during the resting period, and the resource density. These statements are valid irrespective whether the state space is discrete, as for eggs, or continuous, as for satiation level and whether the resource acquisition is prey capture, plant biomass eaten or eggs laid. We have sufficient information on all these parameters for only a very few biological system, and partial information for many more. Aphytis is the only one case for which all the parameters are available, in particular its foraging window: 3 to 8 h, or $10-30 \%$ of a day (Casas 2000, Murdoch et al. 2005). A quantification of the foraging window of mantids in the field is unfortunately wanting. Carabid beetles, for which more information is available on diel activity in the field but much less on their digestion and prey capture rates, also forage for a few hours per day only (e.g. $5.3 \mathrm{~h}$ for Pterostichus coerulescens). Spiders are also reported to have only a few hours to forage (6-8 h, Joern et al. 2006) and this does not account for the necessary overlay with the activity window of their prey. Finally, large herbivores spend only $30 \%$ of their daily time budget foraging (Jeschke and Tollrian 2005). Aphytis is therefore in no way a special case.

\section{Transients might be the rule, not the exception}

As explained in the introduction, the assumption of a stationary consumer population distribution is fundamental to the estimate of the attack rate in the proportional model. Our exploration of the full model, based on experimental data obtained for each variable of interest using the Aphytis parasitoid, allowed the identification of the conditions required to give rise to such a stationary population distribution. They are 1) a high host density and 2) a long foraging time, lasting over eight hours. Under those conditions and given that the oviposition rate is much higher than the egg maturation rate, mature eggs do not build up during the foraging period and the eggs matured during the night can also be laid. Note that the population attains the steady state under these conditions only at the end of the foraging period. These findings are likely to apply to many synovigenic parasitoids with life history traits similar to those of Aphytis. However, the identified conditions are not likely to be often met in the field; indeed, Aphytis forages in fact for a mere 3 to $8 \mathrm{~h}$, or $10-30 \%$ of a day (Casas 2000, Murdoch et al. 2005). The same applies most likely to predators and herbivores, as shown above. Thus, transients might be the rule rather than the exception. 


\section{Defining the functional response}

The concept of the functional response as defined by Metz and van Batenburg depends on the following time-scale separation assumption: prey density does not change appreciably over a relatively large number of prey capture events performed by a single predator (Metz and van Batenburg 1985a). This enables the limit $(t \rightarrow \infty)$ to be taken for the satiation process, as it equilibrates much faster than prey or predator dynamics. Consumer diel foraging cycles provide a new time horizon, the end of the foraging period, and new conditions for the use of such limits: the use of the average capture per day as the predation rate is only possible if the consumer's response time (i.e. the time needed for the distribution of the consumer over the state space to attain equilibrium) is much shorter than the foraging period. The same applies to the number of day/night cycles needed for the consumer population to reach a state of pseudo-equilibrium: this number should represent a much shorter period of time than that associated with life-time or generation. Taking Aphytis as a model, in which females emerge eggless (Collier 1995), the overall concept of functional response and its underlying time-scale separation should be reconsidered. Indeed, recently emerged females needed between two and seven days (i.e. cycles) to reach the ovarian pseudo-equilibrium (Fig. 7A, B), whereas the mean lifespan in the field is of the same order: two to five days according to Heimpel et al. (1997), four days at low host density and fourteen days at high host density according to Murdoch et al. (2005). If a consumer would live forever, one could model its distribution by gluing Markov chains when parameters change abruptly and obtain the stationary distribution. This approach breaks down when the time scale for convergence is of the same order as the lifetime.

Figure 7 also reveals an interesting feature of the statedependent transient responses. The pseudo-steady state is attained more or less quickly, depending on the starting conditions. This is explicitly seen on Fig. 7A and 7B, and is a mere consequence of the within-day processes, described above and illustrated for Mantis on Fig. 7C and 7D. The higher frequency of prey captures at high prey density implies that the past conditions are quickly forgotten, within and between days.

\section{Implications at the population level}

Our focus has been to illuminate the consequences of finite foraging periods on attack rates by parasitoids and predators. These effects are profound and have even broader impact when considering dynamic, interacting populations of consumers and their prey.
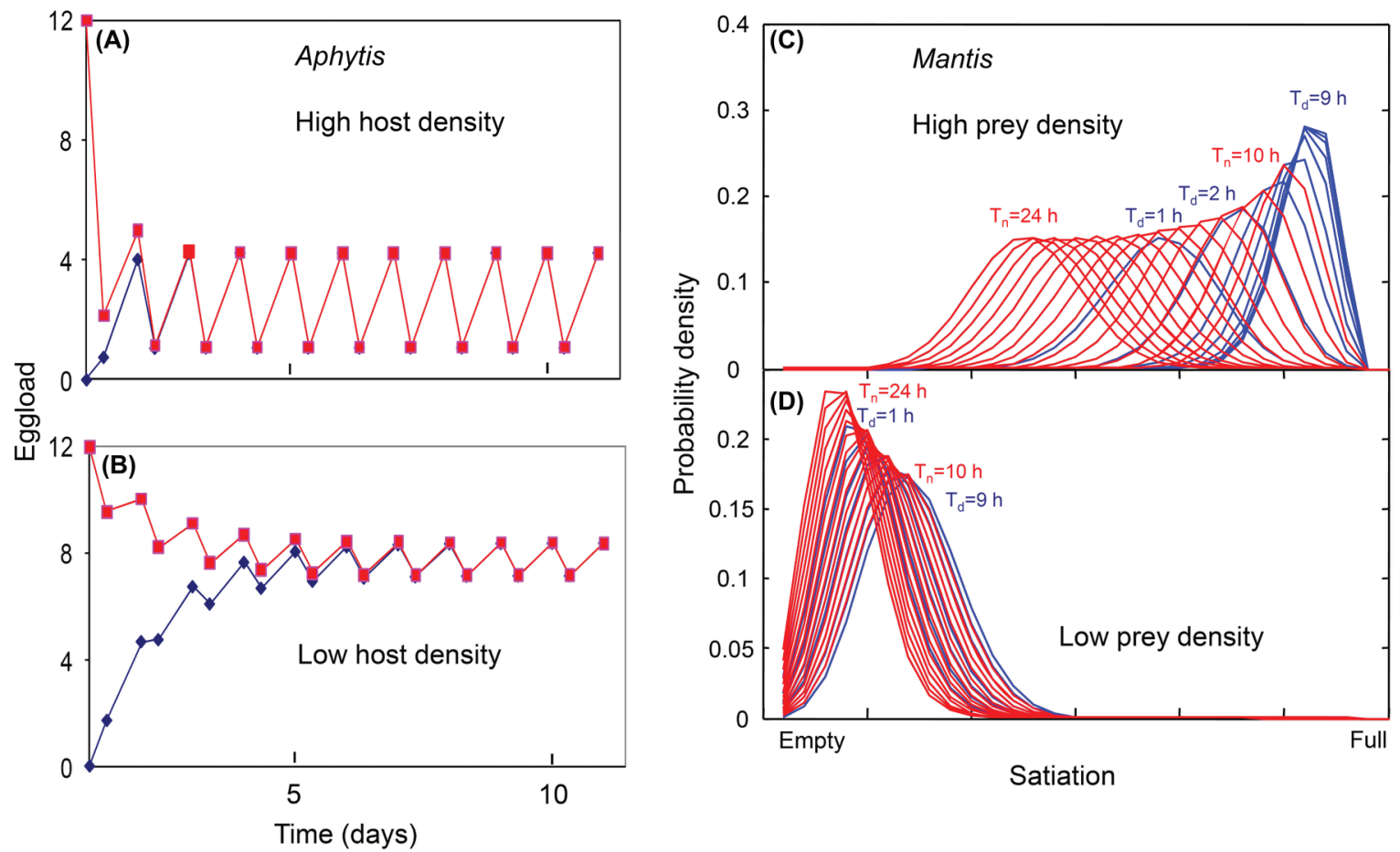

Figure 7. Resource density determines the speed at which transients in the consumer population distribution decay over a lifetime and over a single day. (A) and (B) Mean Aphytis eggload predicted by the stochastic model over many days, as a function of the host density (high in $\mathrm{A}$ and low in B) and starting conditions (eggless in blue and full egg complement of 12 mature eggs in pink). The lowest dip of a cycle is at the end of a foraging period (i.e. day), the highest peak is at the end of a resting period (i.e. night). A pseudo-equilibrium is reached at approximately the end of days 2 and 7, for high and low host densities, respectively. (C) and (D) Daily dynamics of Mantis population distribution, once the pseudo-steady state has been attained, as a function of the host density (high in C and low in D). The hourly distributions during the foraging period (in blue) as well as during the night (in red) are represented. The first as well as the last hour within the foraging period $\left(T_{d}=1 \mathrm{~h}\right.$ and $T_{d}=9 h$, respectively) are marked, as are the first and last hour of the night $\left(T_{n}=10 h\right.$ and $T_{n}=24 h$, respectively). Distributions are far apart when they move quickly, and are stacked once the steady state is attained, a situation occurring only at high resources densities. 

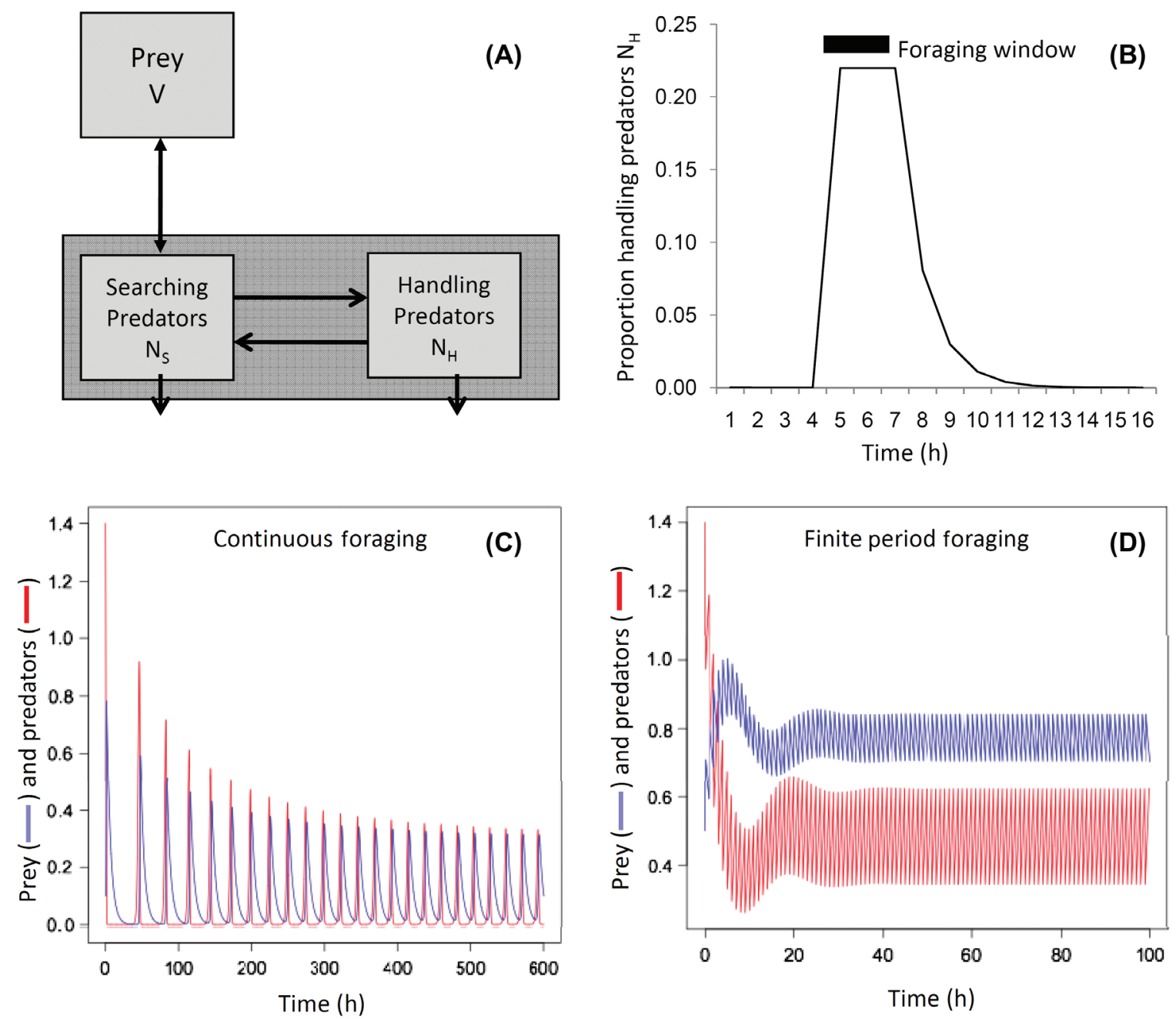

Figure 8. Consequences of a finite period of foraging on the population dynamics of a resource-consumer model related to the RosenzweigMacArthur model. (A) The total population of predators is explicitly identified as searching and handling predators. (B) The number of handling predators is shown to increase without delay with the onset of the foraging window and declines less rapidly after the end of the foraging period. The comparison of the model with continuous $(C)$ and restricted $(D)$ foraging periods shows that the later situation leads to a quicker return time at higher population levels. The model and its parameter values are given in the Appendices.

Restricting the foraging period to a few hours per day and recognizing explicitly the density of handling and searching predators produces (Fig. 8, Appendix 2) significant effects on equilibrium abundances of predators and prey, as well as changes in stability, in a model related to the classic Rozenzweig-MacArthur model. As expected from the quantitative changes in the functional response curves (Fig. 5), restricting foraging periods requires a higher density of predators and prey to achieve the joint equilibrium of resources and consumers, and the system is more stable in the sense of return-time to equilibria. These changes arise from quantitative changes in parameter values, and for these simple phenomenalistic models, do not introduce implicitly new forms of density-dependence. Here, we have illustrated these impacts with one of the most ubiquitous resource-consumer models. In a subsequent paper, we explore the dynamical implications for a variety of parasitoid-host and predatorprey systems using more explicit model formulations that take into account the hybrid mixture of discrete and continuous time-scales. It is only using these explicit formulations that the full-breadth of dynamic implications of adding new time horizons and further multi-scale processes (Getz and Schreiber 1999, Singh and Nisbet 2007) can be revealed.

Acknowledgements - J. A. J. Metz provided detailed feedback on state-dependent models and also made suggestions on numerical techniques for computing transients. We appreciate his support and insight. Olivier Dangles, David Giron, Marlène Goubault, Sylvain Pincebourde, Romain Richard and Christelle Suppo made valuable comments on an earlier version of the manuscript. Support for the research was provided by the CNRS, NCEAS in Santa Barbara and a Le Studium 'Chaire d'excellence' at the University of Tours to EM.

\section{References}

Abrams, P. A. 1982. Functional response of optimal foragers. - Am. Nat. 120: 382-390.

Briggs, C. J. et al. 1995. Dynamical effects of host-feeding in parasitoids. - J. Anim. Ecol. 64: 403-416. 
Casas, J. 2000. Host location and selection in the field. - In: Hochberg, M. E. and Ives, A. R. (eds), Parasitoid population biology. Princeton Univ. Press, pp. 16-26.

Casas, J. et al. 1993. A probabilistic model for the functional response of a parasitoid at the behavioural time scale. - J. Anim. Ecol. 63: 194-204.

Casas, J. et al. 2000. Eggload dynamics and oviposition rate in a wild population of a parasitic wasp. - J. Anim. Ecol. 69: 185-193.

Casas, J. et al. 2004. Parasitoid behaviour: predicting field from laboratory. - Ecol. Entomol. 29: 657-665.

Collier, T. R. 1995. Host feeding, egg maturation, resorption, and longevity in the parasitoid Aphytis melinus (Hymenoptera, Aphelinidae). - Ann. Entomol. Soc. Am. 88: 206-214.

Collier, T. R. et al. 1994. Egg load and the decision to host-feed in the parasitoid Aphytis melinus. - J. Anim. Ecol. 63: 299-306.

Dunlap, J. C. et al. 2009. Chronobiology: time keeping. - Sinauer.

Englund, G. and Leonardsson, K. 2008. Scaling up the functional response for spatially heterogeneous systems. - Ecol. Lett. 11: 440-449.

Fellows, M. D. E. et al. 2007. Foraging behaviour. - In: Jervis, M. A. (ed.), Natural enemies: a practical perspective. Springer, pp. 1-71.

Fernandez-Arhex, V. and Corley, J. C. 2003. The functional response of parasitoids and its implications for biological control. - Biol. Control Tech. 13: 403-413.

Fryxell, J. M. and Lundberg, P. 1997. Individual behaviour and community dynamics. - Chapman \& Hall.

Fryxell, J. M. et al. 2007. Group formation stabilizes predator-prey dynamics. - Nature 449: 1041-1043.

Getz, W. M. and Schreiber, S. J. 1999. Multiple time scales in consumer-resource interactions. - Ann. Zool. Fenn. 36: 11-20.

Gregorini, P. et al. 2008. Review: the interaction of diurnal grazing pattern, ruminal metabolism, nutrient supply and management in cattle. - Prof. Anim. Sci. 24: 308-318.

Guillemette, M. 1998. The effect of time and digestion constraints in common eiders while feeding and diving over blue mussel beds. - Funct. Ecol. 12: 123-131.

Gurney, W. S. C. et al. 1990. The physiological ecology of Daphnia: a dynamic model of growth and reproduction. - Ecology 71 : 716-732.

Hall, S. J. et al. 1995. Inferring feeding patterns from stomach contents data. - J. Anim. Ecol. 64: 39-62.

Heimpel, G. E. et al. 1997. Predation on adult Aphytis parasitoids in the field. - Oecologia 110: 346-352.

Hobbs, N. et al. 2003. Herbivore functional response in heterogeneous environments: a contest among models. - Ecology 84: 666-681.

Holling, C. S. 1966. The functional response of invertebrate predators to prey density. - Mem. Entomol. Soc. Can. 48: 1-87.

Hunter, J. J. 1983. Mathematical techniques of applied probability. - Academic Press.

Jeschke, J. and Tollrian, R. 2005. Predicting herbivore feedings times. - Ethology 111: 187-206.
Jeschke, J. et al. 2002. Predator functional responses: discriminating between handling and digesting prey. - Ecol. Monogr. 72: 95-112.

Joern, A. et al. 2006. Natural history of mass-action in predatorprey models: a case study from wolf spiders and grasshoppers. - Am. Midl. Nat. 156: 52-64.

Keeling, M. J. et al. 2000. Reinterpreting space, time lags, and functional responses in ecological models. - Science 290: 1758-1761.

Kramer, K. and Prins, H. H. T. 2010. Allometric scaling of resource acquisition by ruminants in dynamics and heterogeneous environments. - Ecol. Modell. 221: 2555-2564.

Mangel, M. and Peters, C. S. 1989. Computation of the fitness and functional response of Holling's 'hungry mantid' by the WKB method. - Appl. Math. Lett. 2: 361-365.

Metz, J. A. J. and van Batenburg, F. H. D. 1985a. Holling's 'hungry mantid' model for the invertebrate functional response considered as a Markov process. Part I. The full model and some of its limits. - J. Math. Biol. 22: 209-238.

Metz, J. A. J. and van Batenburg, F. H. D. 1985b. Holling's 'hungry mantid' model for the invertebrate functional response considered as a Markov process. Part II. Negligible handling time. - J. Math. Biol. 22: 239-257.

Morozov, A. Y. 2010. Emergence of Holling type III zooplankton functional response: bringing together field evidence and mathematical modelling. - J. Theor. Biol. 265: 45-54.

Murdoch, W. et al. 2003. Consumer resource dynamics. - Princeton Univ. Press.

Murdoch, W. et al. 2005. Host suppression and stability in a parasitoid-host system: experimental demonstration. - Science 30: 610-613.

Owen-Smith, R. N. 2002. Adaptive herbivore ecology. - Cambridge Univ. Press.

Parthasarathy, P. R. and Lenin, R. B. 2004. Birth and death processes (BDP) models with applications. - Am. Sci. Press.

Rijn, P. C. J. et al. 2005. Is arthropod predation exclusively satiation-driven? - Oikos 109: 101-116.

Sabelis, M. W. 1986. The functional response of predatory mites to the density of twospotted spider mites. - In: Metz, J. A. J. and Diekmann, O. (eds), The dynamics of physiologically structured populations. Springer, pp. 298-321.

Singh, A. and Nisbet, R. 2007. Semi-discrete host-parasitoid models. - J. Theor. Biol. 247: 733-742.

Tschanz, B. et al. 2007. Functional responses: a question of alternative prey and predator density. - Ecology 88: 1300-1308.

Turchin, P. 2003. Complex population dynamics. A theoretical/ empirical synthesis. - Princeton Univ. Press.

van Vianen, A. and van Lenteren, J. C. 1986. The parasite-host relationship between Encarsia formosa Gahan (hymenoptera: Aphelinidae) and Trialeurodes vaporariorum (Homoptera: Aleyrodidae). XV. Oogenesis and oviposition by Encarsia. - J. Appl. Entomol. 102: 130-139.

Van den Meiracker, R. A. F. and Sabelis, M. W. 1999. Do functional responses of predatory arthropods reach a plateau? A case study of Orius insidiosus with western flower thrips as prey. - Entomol. Exp. Appl. 9: 323-329.

Zhang, S. et al. 2010. Functional synchronization of biological ryhtms in a tritrophic systm. - PLoS One 5: e11064. 


\section{Appendix 1}

\section{Comparing the results of Holling's experiments and our predictions}

A comparison of the predictions of the full model with those of Holling's original model, which was fitting his data set very well, leads to four observations. First, the overall shapes of the functional responses are very similar between our simulations and his observations and differ mainly in the values of the plateau. Second, Holling's model invariably predicts higher numbers of prey attacked. Third, the discrepancies are negligible at low prey densities and short foraging windows. All three observations can be explained by noting that Holling starved his mantids, both experimentally and in the model, for $36 \mathrm{~h}$ before running the experiment. They were therefore highly motivated in pursuing and capturing prey, in contrast to our simulated predators which were in a pseudosteady state. This difference shows up over extended period of time and at high prey density. Finally, other differences stem from the processes involved in Holling's original model and in our simplified version, borrowed from Metz and van Batenburg. By starting its experiments with nearly starved predators, Holling positioned them in a region of the satiation axis where handling times are non-negligible, in contrast to our assumption, based on the pseudo-steady state. The dynamics of system in the transient regime is known to depend crucially on the details of the starting conditions.

\section{Appendix 2}

Let us model the resource-consumer interaction with:

$\frac{d V}{d t}=r V\left[1-\frac{V}{K}\right]-\varphi N_{S} V$

With $V$ for the density of the resource, $r$ for its growth rate, $K$ for its carrying capacity and $\varphi$ for the capture rate. The total number of predators, $N$, is the sum of the searching $N_{S}$ and handling predators $N_{H}$

$$
\begin{aligned}
& N=N_{S}+N_{H} \\
& \frac{d N}{d t}=\beta \varphi N_{S} V-\delta N \\
& \frac{d N_{S}}{d t}=\frac{1}{\tau} N_{H}+\beta \varphi N_{S} V-\varphi N_{S} V-\delta N_{S} \\
& \frac{d N_{H}}{d t}=\varphi N_{S} V-\delta N_{H}-\frac{1}{\tau} N_{H}
\end{aligned}
$$

With $\beta$ being the conversion factor, $\delta$ the death rate and $\tau$ the handling time. The parameter values for Fig. 8 are $r=1$, $K=1.4, \varphi=10, \beta=0.5, \delta=0.1$ and $\tau=0.1$. 